\title{
ARTÍGULO ORIGINAL: Diferencias en el Control Metabólico entre Adolescentes y Adultos con Diabetes Mellitus Tipo 1 en el Hospital San Juan De Dios, de Marzo a Agosto 2007.
}

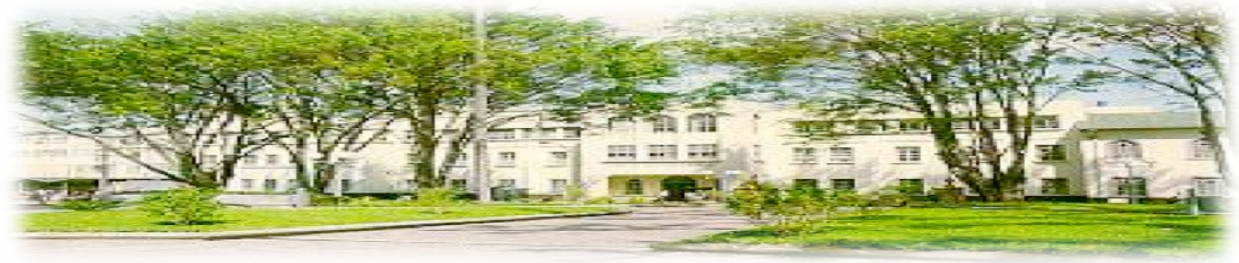

Haspital San quan de Dias, San Jasé, Costa Rica. Fundada en 1845

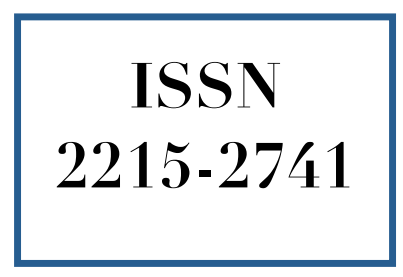

Recibido: $\quad 14 / 01 / 2013$

Aceptado: $\quad$ 22/02/2013

Abner Aguilar Valdés ${ }^{1}$

${ }^{1}$ Médico Especialista en Endocrinología. Asistente del Servicio de Endocrinología. Sección de Medicina. Hospital San Juan de Dios.

\section{RESUMEN}

Introducción: El debut y/o evolución de la diabetes mellitus tipo 1 se presenta con frecuencia durante la adolescencia, etapa caracterizada por la aspiración a la independencia y en ocasiones a conductas de riesgo. El objetivo del estudio fue comparar el control glicémico y características epidemiológicas entre adolescentes y adultos diabéticos tipo 1. Métodos: Se realizó un estudio observacional transversal con 99 diabéticos tipo 1 , valorando variables de la historia clínica, de laboratorio (control glicémico, excreción urinaria de albúmina, perfil lipídico) y complicaciones crónicas micro y macrovasculares de los sujetos. Se definió como adolescentes aquellos sujetos menores o iguales a 18 años y adultos aquellos mayores de 18 años. Se efectuó un análisis comparativo de las variables entre el grupo de adolescentes $(\mathrm{n}=34)$ y el de adultos $(\mathrm{n}=65)$. Resultados: La edad promedio de los sujetos fue de 25.0土9.9 años, con una duración promedio de la diabetes de $12.8 \pm 8.6$ años. Hubo diferencias estadísticamente significativas entre adolescentes y adultos diabéticos tipo 1 en las siguientes variables: edad de diagnóstico $(\mathrm{P}=0.005)$, edad de referencia $(\mathrm{P}=0.002)$, índice de masa corporal $(\mathrm{P}<0.001)$, presencia de dislipidemia $(\mathrm{P}<0.001)$, retinopatía $(\mathrm{P}=0.018)$ y nefropatía diabéticas $(\mathrm{P}=0.001)$, requerimientos de insulina $(\mathrm{P}=0.041)$, uso de inhibidores de la ECA $(\mathrm{P}=0.001)$ y estatinas $(\mathrm{P}<0.001)$ y niveles de $\mathrm{HbAlc}$ $(\mathrm{P}=0.009)$ y de triglicéridos $(\mathrm{P}=0.038)$. El control glicémico promedio fue significativamente peor en el grupo de adolescentes $(\mathrm{HbA} 1 \mathrm{c}=9.35 \pm 2.26 \%)$ en comparación con el de adultos $(\mathrm{HbA} 1 \mathrm{c}=8.23 \pm 1.81 \%)$, sin embargo ésto no se tradujo en mayor prevalencia de complicaciones crónicas al momento del análisis, en relación con el grupo de adultos. Conclusiones: La adolescencia puede ejercer un efecto significativo y deletéreo en el control metabólico de la diabetes mellitus tipo 1 en comparación con la etapa adulta de la vida. 


\section{PALABRAS CLAVE}

Diabetes Mellitus Tipo 1. Adolescencia. Macrovascular. Microvascular.

\section{ABSTRACT}

Background: Debut and/or progression of type 1 diabetes mellitus often occurs during adolescence, a stage characterized by the desire for independence and sometimes risky behaviors. The aim of the study was to compare glycemic control and epidemiology in adolescents and adults with type 1-Diabetes. Methods: We performed an observational cross-sectional study with 99 type 1 diabetics, assessing variables of medical history, laboratory results (glycemic control, urinary albumin excretion, lipid profile) and chronic micro and macrovascular complications. Adolescents were defined as subjects aged 18 years or less and adults, subjects over 18 . We performed a comparative analysis of the variables between the adolescent group $(n=34)$ and adult group $(n=65)$ of the study. Results: The average age of the subjects was $25.0 \pm 9.9$ years, with an average duration of diabetes of $12.8 \pm$ 8.6 years. There were statistically significant differences between adolescents and adults with type 1 diabetes in the following variables: age at diagnosis $(\mathrm{P}=0.005)$, baseline age $(\mathrm{P}=0.002)$, body mass index $(\mathrm{P}<0.001)$, presence of dyslipidemia $(\mathrm{P}<0.001)$, diabetic retinopathy $(\mathrm{P}=0.018)$ and diabetic nephropathy $(\mathrm{P}=0.001)$, insulin requirements $(\mathrm{P}=0.041)$, use of $\mathrm{ACE}$ inhibitors $(\mathrm{P}=0.001)$ and statins $(\mathrm{P}<0.001)$, HbA1c levels $(\mathrm{P}=0.009)$ and triglycerides $(\mathrm{P}=0.038)$. The average glycemic control was significantly worse in the adolescent group $(\mathrm{HbA} 1 \mathrm{c}=9.35 \pm 2.26 \%)$ compared with adults $(\mathrm{HbA} 1 \mathrm{c}=8.23 \pm 1.81 \%)$, however, this did not translate into increased prevalence of chronic complications at the time when analysis was made. Conclusions: Adolescence can exert a significant and deleterious effect on the metabolic control of type 1 diabetes mellitus compared with the adult stage of life.

\section{KEY WORDS}

Type 1 Diabetes Mellitus. Adolescence. Macrovascular. Microvascular.

\section{INTRODUCCIÓN}

La diabetes mellitus tipo 1 es un trastorno metabólico caracterizado por la destrucción inmunitaria o idiopática de las células beta del páncreas, que comparte el fenotipo de la hiperglicemia, siendo ésta última uno de los factores etiopatogénicos en el desarrollo de complicaciones agudas y crónicas de la enfermedad $^{(1)}$. La prevalencia mundial de esta patología ha aumentado de forma lineal a partir de la segunda mitad del siglo XX y principalmente en las últimas 2 décadas, con mayor incremento en la incidencia en el grupo etario menor a 5 años, en comparación con otros grupos $^{(2)}$. A pesar del incremento en la frecuencia de la diabetes tipo 1, ésta constituye menos del $10 \%$ de las causas de diabetes a nivel mundial. La edad de presentación típica de la diabetes tipo 1 es antes de los 20 años, siendo el $80 \%$ de los casos antes de los $30^{(1)}$. Estos datos epidemiológicos revelan el hecho de que una notable proporción de diabéticos tipo 1 presentan su debut, adaptación a y convivencia diaria con la enfermedad durante el período de la adolescencia. Ésta constituye una etapa de la vida que establece una transición entre la niñez y la edad adulta y se caracteriza por la aparición de cambios físicos y psicológicos espectaculares. El componente psicológico de la adolescencia abarca cambios en las siguientes 5 esferas: desarrollo de pensamiento abstracto, forjamiento de identidad personal y sexual, incremento en nivel de socialización, independencia respecto de la familia y establecimiento de un sistema de valores $^{(3)}$. Algunos autores definen la adolescencia desde el punto de vista temporal, como el lapso de tiempo que transcurre entre los 10 y 19 años. Los adolescentes constituyen un grupo poblacional con tendencia a los comportamientos y conductas de riesgo, en relación con un sentimiento de inmortalidad frente a accidentes y patologías previamente establecidos o desarrollados durante el curso de esta etapa de la vida ${ }^{(3)}$.

La presencia de enfermedades crónicas durante la adolescencia puede generar sentimientos de angustia, negación, falta de conciencia de enfermedad y conflictos familiares que a su vez, pueden condicionar un deterioro en el manejo y seguimiento multidisciplinario de la patología por factores tales como: asistencia irregular a citas de seguimiento médico, debilitamiento en la 
relación médico-paciente, falta de adherencia a la terapia, falta de acceso a atención médica especializada y limitaciones en las esferas educativa y económica del paciente y su familia.

En Estados Unidos, la diabetes tipo 1 es una de las patologías crónicas más frecuentes entre niños y adolescentes. Estudios previos han revelado que hasta el $40 \%$ de los adolescentes diabéticos tipo 1 desarrollan complicaciones crónicas microvasculares de la enfermedad, siendo el riesgo mayor en aquellos con diabetes pobremente controlada ${ }^{(4)}$. Dentro de los factores que se han relacionado con deterioro en el control glicémico en adolescentes diabéticos tipo 1, se describen la falta de adherencia al tratamiento, el temor frente a los episodios de hipoglicemia y la coexistencia de ciertas patologías psiquiátricas como depresión, trastornos de ansiedad y trastornos alimentarios ${ }^{(5)}$.

El presente estudio se realizó con el objetivo de comparar el control metabólico y la prevalencia de complicaciones crónicas de la enfermedad entre el grupo de adolescentes y adultos diabéticos tipo 1 de la Consulta Externa del Hospital San Juan de Dios, San José, Costa Rica, año 2007.

\section{DISEÑO DEL ESTUDIO}

Estudio observacional transversal. Entre los meses de marzo y agosto del año 2007 se revisó los expedientes de todos los pacientes con diagnóstico previo de diabetes tipo 1 de la Consulta Externa de Endocrinología del Hospital San Juan de Dios (HSJD), un hospital universitario localizado en San José, la capital de Costa Rica. Los expedientes fueron revisados el día en el que el paciente asistía a su cita de seguimiento médico a cargo del endocrinólogo de dicho centro hospitalario. Se definió como punto de corte la edad de 18 años para distinguir adolescentes ( $\leq 18$ años) de adultos ( $>18$ años).

Se registró datos clínicos y de laboratorio para cada paciente incluido en el estudio. Las variables clínicas analizadas fueron divididas en datos de la anamnesis y datos del examen físico. Las primeras incluyeron: ficha de identificación, edad de diagnóstico de diabetes tipo 1 , edad de referencia al HSJD, años de evolución de la enfermedad, antecedentes personales patológicos $\mathrm{y}$ heredofamiliares y tratamiento farmacológico actual. En el caso del tratamiento, se determinó el tipo de esquema de insulina utilizado por cada paciente (esquema intensificado con insulina simple y NPH, con análogos de insulina de acción ultrarrápida en combinación con análogos de acción prolongada o combinaciones de los anteriores), esquema mixto dividido, dos dosis de insulina NPH, tres dosis de insulina (combinando simple con NPH) y la dosis total diaria de insulina. Además se anotó los otros fármacos utilizados por el paciente y las dosis empleadas.

El peso, la talla, la presión arterial y la circunferencia abdominal fueron determinados y registrados el día de la cita del paciente, datos que constituyeron las variables del examen físico. Las mediciones determinadas fueron obtenidas en igualdad de condiciones para cada paciente del estudio: todos fueron medidos y pesados descalzos, en la misma báscula, por el mismo explorador; la presión arterial se determinó con el mismo esfigmomanómetro por el mismo explorador para todos los pacientes y la circunferencia abdominal se midió mediante una cinta métrica flexible inextensible por un único explorador. La medición de la circunferencia abdominal fue antecedida por el consentimiento informado verbal de cada paciente.

Las variables de laboratorio registradas fueron: glicemia en ayunas (en mg/dl), HbAlc (en \%), microalbuminuria (en $\mu \mathrm{g} / \mathrm{mg}$ creatinina), proteinuria ( $\mathrm{mg} / 24$ horas), perfil lipídico (en $\mathrm{mg} / \mathrm{dl}$ : colesterol total, LDL colesterol, HDL colesterol, triglicéridos) y transaminasas (en UI/l: AST y ALT). Se anotó únicamente los datos de laboratorio más recientes, correspondientes a la toma de muestras de laboratorio para ser analizados el día de la cita médica del paciente entre los meses de marzo y agosto 2007.

Finalmente, fureon registradas las complicaciones crónicas micro y macrovasculares y sus estadios para cada paciente. Esta información fue obtenida a partir del expediente clínico. Las complicaciones microvasculares se clasificaron de la siguiente forma: retinopatía diabética (no proliferativa $v s$ proliferativa), nefropatía diabética (estadio I: microalbuminuria reversible; estadio II: hiperfiltración glomerular y renomegalia; estadio III: microalbuminuria/nefropatía diabétia incipiente; estadio IV: macroalbuminuria/nefropatía diabética abierta; 
estadío V: insuficiencia renal terminal) y neuropatía diabética (somática, visceral o autonómica).

La determinación de retinopatía diabética y sus estadios estuvo a cargo de un oftalmólogo y no del endocrinólogo tratante. Se definió micro y macroalbuminuria como tasas de excreción urinaria de albúmina entre 30 y $300 \mu \mathrm{g} / \mathrm{mg}$ creatinina y mayor de $300 \mu \mathrm{g} / \mathrm{mg}$ creatinina, respectivamente. Las complicaciones crónicas macrovasculares registradas fueron: enfermedad cardiovascular y enfermedad arterial periférica con manifestaciones clínicas: amputaciones no traumáticas de extremidades inferiores.

\section{Procesamiento de datos}

El análisis de las variables del estudio se realizó mediante el programa SPSS 14.0 para Windows.

\section{RESULTADOS}

\section{Datos de la Anamnesis}

Fueron incluídos los datos de 99 diabéticos tipo 1. La edad promedio al momento del diagnóstico de la diabetes fue de $12.2 \pm 6.4$ años, con una edad de referencia promedio al HSJD de $16.9 \pm$ 6.2 años. La edad promedio al momento del análisis de los datos (29/08/07) fue de $25.0 \pm 9.9$ años. La evolución promedio de la enfermedad fue de $12.8 \pm 8.6$ años. La separación de los sujetos del estudio por grupos etarios, reveló la presencia de 34 adolescentes (34.3\%) y 65 adultos (65.7\%). Hubo diferencias estadísticamente significativas en la edad de diagnóstico de diabetes tipo $1(\mathrm{P}=0.005)$ y edad de referencia al HSJD $(\mathrm{P}=0.002)$ entre adolescentes y adultos, siendo las edades más tempranas en los primeros (cuadro 3). En cuanto a su distribución por género, más de la mitad de la población del estudio correspondió a mujeres, con un total de $57(57.6 \%)$.

Los antecedentes personales patológicos de importancia más frecuentes, en orden decreciente fueron: dislipidemia $(33.3 \%)$, tiroidopatías $(15.2 \%)$, hipertensión arterial (10\%) y depresión (4\%) (cuadros 1 y 2). La dislipidemia fue casi exclusiva de los diabéticos tipo 1 adultos (97\%) en comparación con los adolescentes (3\%); casi la mitad de los adultos asoció dislipidemia y menos de un $3 \%$ de los adolescentes $(\mathrm{P}<0.001)$ (cuadro 3). La patología tiroidea más frecuente fue el hipotiroidismo primario aislado $(n=9)$, seguida de hipotiroidismo subclínico $(n=2)$. Otras patologías tiroideas registradas fueron: tiroiditis de Hashimoto, hipertiroidismo primario, hipotiroidismo y cáncer papilar de tiroides y nódulo tiroideo eufuncionante (cuadro 2). No hubo diferencias estadísticamente significativas en la prevalencia de tiroidopatías entre la población de adolescentes y adultos diabéticos tipo 1 (cuadro 3). Se documentó hipertensión arterial en 8 adultos y 1 adolescente diabéticos tipo 1, diferencia que no alcanzó significancia estadística (cuadro 3). De los 99 pacientes, 4 tenían el diagnóstico previo de depresión y recibían control en psiquiatría.

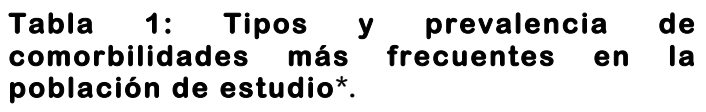

\begin{tabular}{|c|c|c|}
\hline Diagnóstico & \multicolumn{2}{c|}{ Frecuencia $\begin{array}{c}\text { Porcentaje } \\
\text { del total }\end{array}$} \\
\hline Dislipidemia & 33 & $\mathbf{3 3 , 3 \%}$ \\
\hline Tiroidopatías & 15 & $\mathbf{1 5 , 2 \%}$ \\
\hline $\begin{array}{c}\text { Hipertensión } \\
\text { arterial }\end{array}$ & 9 & $\mathbf{9 , 1 \%}$ \\
\hline Gastritis & 4 & $\mathbf{4 , 0 \%}$ \\
\hline Asma & 4 & $\mathbf{4 , 0 \%}$ \\
\hline Depresión & 4 & $\mathbf{4 , 0 \%}$ \\
\hline Epilepsia & 2 & $\mathbf{2 , 0 \%}$ \\
\hline $\begin{array}{c}\text { Síndrome de } \\
\text { Down }\end{array}$ & 2 & $\mathbf{2 , 0 \%}$ \\
\hline \hline Total & $\mathbf{7 3}^{\mathbf{a}}$ & $\mathbf{7 3 , 7 \%}{ }^{\mathbf{a}}$ \\
\hline
\end{tabular}

* Los datos se basan en el grupo de 99 diabéticos tipo 1 de la Consulta Externa del HSJD; ${ }^{a}$ las comorbilidades no son mutuamente excluyentes, por lo que un paciente podía presentar una o varias de ellas de forma simultánea. Fuente: Base de datos del trabajo.

El antecedente heredofamiliar más prevalente fue la diabetes mellitus tipo 2, presente en poco menos de la mitad de la población de estudio $(47.5 \%)$. En orden decreciente de frecuencia le siguieron: diabetes mellitus tipo 1 (12.1\%), asma bronquial (6\%), hipertensión arterial (4\%) y cardiopatías $(4 \%)$.

En relación con el esquema de tratamiento insulínico utilizado por los sujetos del estudio, cerca de tres cuartas partes emplearon un esquema intensificado $(74.7 \%$; de los cuales $69.7 \%$ insulina simple y NPH y $5 \%$ insulina 
simple y glargina), el $16.1 \%$ un esquema mixto dividido y el $9,1 \%$ un esquema con dos dosis de NPH. Hubo diferencias estadísticamente significativas en los requerimientos de insulina valorados en unidades internacionales por unidad de peso por día, entre el grupo de adolescentes y el de adultos del estudio, con mayores requerimientos por parte de los primeros $(\mathrm{P}=0.041)$ (cuadro 3).

Tabla 2: Tipos y prevalencia de patologías tiroideas en la población de estudio*.

\begin{tabular}{|c|c|c|}
\hline Diagnóstico & Frecuencia & $\begin{array}{c}\text { Porcentaje } \\
\text { del total }\end{array}$ \\
\hline $\begin{array}{l}\text { Hipotiroidismo } \\
\text { primario }\end{array}$ & 9 & $9,1 \%$ \\
\hline $\begin{array}{l}\text { Hipotiroidismo } \\
\text { subclínico }\end{array}$ & 2 & $2,0 \%$ \\
\hline $\begin{array}{c}\text { Hipotiroidismo } \\
\text { primario y CA } \\
\text { papilar de tiroides* }\end{array}$ & 1 & $1,0 \%$ \\
\hline $\begin{array}{l}\text { Nódulo tiroideo } \\
\text { eufuncionante }\end{array}$ & 1 & $1,0 \%$ \\
\hline $\begin{array}{l}\text { Tiroiditis de } \\
\text { Hashimoto }\end{array}$ & 1 & $1,0 \%$ \\
\hline $\begin{array}{l}\text { Hipertiroidismo } \\
\text { primario }\end{array}$ & 1 & $1,0 \%$ \\
\hline Total & 15 & $15,2 \%$ \\
\hline
\end{tabular}

* Los datos se basan en el grupo de 99 diabéticos tipo 1 de la Consulta Externa del HSJD; CA papilar denota carcinoma papilar de tiroides.

Fuente: Base de datos del trabajo.

Dentro de los otros tratamientos farmacológicos utilizados por los pacientes, los tres fármacos mayormente empleados en orden decreciente de frecuencia fueron: inhibidores de la ECA (IECAs) (36.4\%), estatinas $\quad(30.3 \%) \quad y$ antagonistas del receptor de angiotensina II subtipo I (ARA II) (11.1\%). De los pacientes utilizando IECAs, la mayoría fueron adultos $(88.9 \%)(\mathrm{P}<0.001)$ y casi la mitad de los adultos estaba recibiendo un fármaco perteneciente a este grupo terapéutico, en su mayoría: enalapril (cuadro 3). No hubo diferencias significativas en cuanto al uso de ARA II por parte de los dos grupos. El uso de estatinas fue significativamente mayor en el grupo de adultos $(n=29)$ en comparación con el grupo de adolescentes $(n=1)$ $(\mathrm{P}<0.001)$ (cuadro 3). La estatina mayormente utilizada fue la lovastatina.

\begin{tabular}{|c|c|c|c|}
\hline Criterio & $\begin{array}{c}\text { Adolescentes } \\
(\mathbf{n}=\mathbf{3 4})\end{array}$ & $\begin{array}{c}\text { Adultos } \\
(n=65)\end{array}$ & $\mathbf{P}$ \\
\hline HbA1c & $9.35 \pm 2.26$ & $8.23 \pm 1.81$ & 0.009 \\
\hline IMC & $22.64 \pm 2.89$ & $25.89 \pm 3.64$ & $<0.001$ \\
\hline $\begin{array}{c}\text { Dosis insulina } \\
\text { / kg peso }\end{array}$ & $1.14 \pm 0.37$ & $0.98 \pm 0.34$ & 0.041 \\
\hline Tiroidopatía & $14.7 \%$ & $15.4 \%$ & 0.589 \\
\hline HTA & $2.9 \%$ & $12.3 \%$ & 0.118 \\
\hline Dislipidemia & $2.9 \%$ & $49.2 \%$ & $<0.001$ \\
\hline Retinopatía & $0 \%$ & $13.8 \%$ & 0.018 \\
\hline Nefropatía & $17.6 \%$ & $52.3 \%$ & 0.001 \\
\hline Neuropatía & $2.9 \%$ & $3.1 \%$ & 0.729 \\
\hline $\begin{array}{c}\text { Edad de } \\
\text { diagnóstico }\end{array}$ & $\begin{array}{l}9.71 \pm 3.87 \\
\text { años }\end{array}$ & $\begin{array}{c}13.48 \pm 7.11 \\
\text { años }\end{array}$ & 0.005 \\
\hline $\begin{array}{l}\text { Edad de } \\
\text { referencia }\end{array}$ & $14.18 \pm 1.08$ & $18.29 \pm 7.30$ & 0.002 \\
\hline Uso de IECAs & $11.8 \%$ & $49.2 \%$ & $<0.001$ \\
\hline $\begin{array}{c}\text { Uso } \\
\text { concomitante } \\
\text { de ARA II }\end{array}$ & $5.9 \%$ & $13.8 \%$ & 0.198 \\
\hline $\begin{array}{c}\text { Uso } \\
\text { concomitante } \\
\text { de estatinas }\end{array}$ & $2.9 \%$ & $44.6 \%$ & $<0.001$ \\
\hline $\begin{array}{l}\text { Presión } \\
\text { arterial } \\
\text { sistólica }\end{array}$ & $100.35 \pm 27.15$ & $\begin{array}{c}109.05 \pm 18.5 \\
5\end{array}$ & 0.066 \\
\hline $\begin{array}{c}\text { Presión } \\
\text { arterial } \\
\text { diastólica }\end{array}$ & $70.88 \pm 11.64$ & $70.79 \pm 10.13$ & .969 \\
\hline $\begin{array}{l}\text { Niveles de } \\
\text { proteinuria }\end{array}$ & $148.33 \pm 37.02$ & $\begin{array}{c}216.91 \pm 228 \\
21\end{array}$ & 0.613 \\
\hline $\begin{array}{c}\text { Niveles de } \\
\text { colesterol total }\end{array}$ & $198.18 \pm 51.86$ & $\begin{array}{c}182.57 \pm 38.5 \\
2\end{array}$ & .97 \\
\hline $\begin{array}{l}\text { Niveles de } \\
\text { LDL }\end{array}$ & $111.92 \pm 30.60$ & $\begin{array}{c}111.03 \pm 31.1 \\
3\end{array}$ & 0.896 \\
\hline $\begin{array}{l}\text { Niveles de } \\
\text { HDL }\end{array}$ & $50.00 \pm 12.36$ & $51.18 \pm 12.41$ & 0.659 \\
\hline $\begin{array}{l}\text { Niveles de } \\
\text { triglicéridos }\end{array}$ & $\begin{array}{c}157.69 \pm 144.7 \\
6\end{array}$ & $\begin{array}{c}108.78 \pm 84.2 \\
0\end{array}$ & 0.038 \\
\hline $\begin{array}{c}\text { Porcentaje de } \\
\text { pacientes con } \\
\text { HbA1c menor } \\
\text { a } 6.5 \%\end{array}$ & $11.8 \%$ & $9.2 \%$ & 0.470 \\
\hline $\begin{array}{c}\text { Porcentaje de } \\
\text { pacientes con } \\
\text { HbA1c menor } \\
\text { a } 7 \%\end{array}$ & $14.7 \%$ & $24.6 \%$ & 0.189 \\
\hline $\begin{array}{c}\text { Porcentaje de } \\
\text { pacientes con } \\
\text { LDL menor a } \\
100 \mathrm{mg} / \mathrm{dl}\end{array}$ & $41.2 \%$ & $35.4 \%$ & 0.536 \\
\hline $\begin{array}{l}\text { Porcentaje de } \\
\text { pacientes que } \\
\text { alcanzan PAS } \\
\quad<130\end{array}$ & $94.1 \%$ & $90.8 \%$ & 0.584 \\
\hline $\begin{array}{c}\text { Porcentaje de } \\
\text { pacientes que } \\
\text { alcanzan PAD } \\
<80\end{array}$ & $91.2 \%$ & $90.8 \%$ & 0.530 \\
\hline
\end{tabular}

Tabla 3: Comparación de los Resultados de Variables Clínicas y de Laboratorio entre los Grupos de Adolescentes y Adultos.

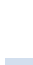




\section{Datos del Examen Físico}

El registro de las variables del examen físico de peso y talla permitió la determinación del índice de masa corporal (IMC) de los sujetos del estudio. El promedio de IMC fue de $24.7 \pm 3.7$ $\mathrm{kg} / \mathrm{m} 2$, lo cual aún se encuentra dentro del límite superior de normalidad para esta medida, sin embargo presenta traslape con el rango de sobrepeso (este último definido como un IMC entre 25 y $30 \mathrm{~kg} / \mathrm{m} 2)$. El análisis del IMC según el grupo etario reveló diferencias estadísticamente significativas. Los IMC promedio para el grupo de adolescentes y adultos fueron de $22.6 \pm 2.9 \mathrm{~kg} / \mathrm{m} 2$ y $25.9 \pm 3.6 \mathrm{~kg} / \mathrm{m} 2$, respectivamente, lo cual sugiere que la mayoría de adolescentes se ubicaron dentro de valores normales y la mayoría de adultos cumplieron criterios de sobrepeso, $\quad(\mathrm{P}<0.001)$. La circunferencia abdominal promedio fue de $83.9 \pm 10.9 \mathrm{~cm}$.

El análisis de los datos concernientes a las cifras tensionales de los diabéticos tipo 1, reveló promedios de presión arterial sistólica (PAS) de $106.0 \pm 22.2 \mathrm{mmHg} y$ de presión arterial diastólica (PAD) de $70.8 \pm 10.6 \mathrm{mmHg}$. No hubo diferencias significativas en las cifras de PAS y PAD entre adolescentes y adultos $(\mathrm{P}=0.066 \mathrm{y}$ $\mathrm{P}=0.969$, respectivamente. (cuadro 3 ).

\section{Datos de Laboratorio}

La HbA1c promedio fue de $8.6 \pm 2.0 \%$. El análisis del control metabólico de acuerdo con el grupo etario reveló diferencias estadísticamente significativas. Los promedios de HbAlc para adolescentes y adultos fueron de $9.4 \pm 2.3 \%$ y $8.2 \pm 1.8 \%$, respectivamente $(\mathrm{P}=0.009)$ (cuadro 3$)$.

En relación con el perfil de lípidos, no hubo diferencias estadísticamente significativas entre adolescentes y adultos en los valores promedio de colesterol total, LDL colesterol y HDL colesterol (cuadro 3). Los niveles promedio de estas variables fueron, respectivamente, $187.9 \pm 43.9 \mathrm{mg} / \mathrm{dl}, \quad 111.3 \pm 30.8 \mathrm{mg} / \mathrm{dl} \quad \mathrm{y}$ $50.8 \pm 12.34 \mathrm{mg} / \mathrm{dl}$. Los niveles promedio de triglicéridos fueron de $125.4 \pm 110.4 \mathrm{mg} / \mathrm{dl}$, siendo significativamente mayores en el grupo de adolescentes en comparación con el grupo de adultos diabéticos tipo $1(\mathrm{P}=0.038)$ (cuadro 3$)$.

De los 99 pacientes del estudio, los datos de albuminuria de 57 de ellos (57.6\% del total) estuvieron disponibles para su análisis; de los cuales 22 fueron de adolescentes y 35 del grupo de adultos. Los niveles promedio de albuminuria fueron de $22.7 \pm 34.7 \mu \mathrm{g} / \mathrm{mg}$ creatinina y no hubo diferencias significativas entre los dos grupos. Estas cifras de excreción urinaria de albúmina se encuentran dentro del rango de normoalbuminuria, con traslape con rangos de microalbuminuria.

El registro de los valores de proteinuria 24 horas estuvo disponible para 29 de los 99 pacientes (29.3\% del total; 3 adolescentes y 26 adultos). El valor promedio de proteinuria fue de $209.8 \pm 216.9 \mathrm{mg} / 24$ horas, $\sin$ diferencias significativas entre adolescentes $y$ adultos $(\mathrm{P}=0.613)$ (cuadro 3).

\section{Pacientes alcanzando metas de tratamiento}

La meta de control glicémico de HbA1c inferior a $6.5 \%$ fue alcanzada por 10 de los 99 pacientes $(10.1 \%$ del total). No hubo diferencias significativas en el porcentaje de pacientes logrando esta meta entre adolescentes $(n=4)$ y adultos $(n=6)(P=0.470)$ (cuadro 3$)$. El 21.2\% de los pacientes tuvo una HbAlc inferior a 7\% $(n=21$; adolescentes $n=5$; adultos $n=16)$. No hubo diferencias significativas en el porcentaje de sujetos alcanzando esta meta entre adolescentes y adultos ( $\mathrm{P}=0.189)$ (cuadro 3 ).

La meta de LDL colesterol inferior a $100 \mathrm{mg} / \mathrm{dl}$ fue alcanzada por 37 pacientes $(37.4 \%$ del total), de los cuales la mayoría $(62.2 \%)$ fueron adultos $(n=23)$. No hubo diferencias en el porcentaje de sujetos alcanzando esta meta entre adolescentes y adultos $(\mathrm{P}=0.536)$ (cuadro 3 ).

El $91.9 \% \quad(n=91)$ de los pacientes lograron la meta de PAS inferior a $130 \mathrm{mmHg}$, sin diferencias en el porcentaje de sujetos alcanzando esta meta entre adolescentes y adultos $(\mathrm{P}=0.584)$ (cuadro 3$)$. El objetivo de PAD inferior a $80 \mathrm{mmHg}$ se registró en el $90.9 \%$ de los pacientes $(\mathrm{n}=90)$, sin diferencias significativas entre ambos grupos $(\mathrm{P}=0.530)$ (cuadro 3).

\section{Complicaciones Crónicas Microvasculares}

La prevalencia de complicaciones crónicas microangiopáticas en orden decreciente de 
frecuencia fue: nefropatía diabética $(40.4 \%$ del total), retinopatía diabética $(9.1 \%$ del total) y neuropatía diabética (3.0\% del total). Más de la mitad de la población adulta presentó nefropatía diabética (52.3\%) en comparación con el 17.6\% de los adolescentes, diferencia que fue estadísticamente significativa $(\mathrm{P}=0.001)$ (cuadro 3).

La presencia de retinopatía diabética fue exclusiva de la población adulta, presente en un total de 9 sujetos; diferencia que alcanzó significancia estadística en relación con el grupo de adolescentes $(\mathrm{P}=0.018)$.

Se documentó neuropatía diabética en un total de tres sujetos, de los cuales dos fueron adultos. No hubo diferencias significativas entre adolescentes y adultos en la prevalencia de esta complicación $(\mathrm{P}=0.729)$ (cuadro 3).

\section{Complicaciones Macrovasculares}

Crónicas

Ninguno de los sujetos del estudio tenía evidencia de enfermedad cardiovascular en alguna de sus formas o amputaciones no traumáticas de extremidades inferiores.

\section{DISCUSIÓN}

El principal objetivo de este estudio fue establecer un análisis comparativo del control metabólico y características epidemiológicas entre el grupo de adolescentes y el de adultos diabéticos tipo 1 de la Consulta Externa del HSJD. A pesar de que la edad promedio de la población fue relativamente joven ( $25 \pm 9.9$ años), la proporción adultos:adolescentes fue de 1.9:1. El hallazgo más sobresaliente del estudio fue la demostración de diferencias estadísticamente significativas entre ambos grupos en un total de 10 variables: edad de diagnóstico, edad de referencia, presencia de dislipidemia, retinopatía y nefropatía diabéticas, requerimientos de insulina, uso de IECAs y estatinas y niveles de HbAlc y de triglicéridos.

El diseño del estudio no permitió establecer de forma objetiva los factores por los cuales se presentaron las diferencias previamente nombradas entre los 2 grupos, sin embargo, existe evidencia a partir de investigaciones previas, de que algunas de las mismas, incluyendo las diferencias en el control glicémico y en el desarrollo de complicaciones crónicas de la enfermedad, pueden ser atribuidas a factores psicosociales ${ }^{(4-6,8-10)}$.

La variable de la anamnesis edad de referencia, es en realidad dependiente tanto de la edad de diagnóstico de la diabetes tipo 1 como de las políticas individuales de referencia de cada centro de atención en salud. Lo anterior explica, al menos parcialmente, el hecho de que los adultos fueran referidos con edades más avanzadas a la Consulta Externa de Endocrinología del HSJD, pues el diagnóstico de la diabetes tipo 1 se estableció de forma más tardía en los mismos, en comparación con el grupo de adolescentes. Resulta intrigante el hallazgo de que el diagnóstico de la enfermedad se estableciera significativamente más temprano en los adolescentes en comparación con los adultos. Varias investigaciones previas han revelado no sólo un incremento marcado en la incidencia de la diabetes tipo 1 durante la segunda mitad del siglo $\mathrm{XX}^{(2,16)}$, sino un mayor aumento en la incidencia de la enfermedad en el grupo etario de 0 a 4 años, en relación con diabéticos tipo 1 de edades mayores ${ }^{(16-18)}$.

El estudio demostró una mayor prevalencia de complicaciones crónicas microvasculares en el grupo de adultos en comparación con el de adolescentes. Los principales factores involucrados en el desarrollo y progresión de dichas complicaciones son: duración de la enfermedad, control glicémico y control de la presión arterial. El control de la presión arterial no fue distinto entre los dos grupos y más del $90 \%$ de los pacientes alcanzaron la meta de tratamiento de PA menor a 130/80 mmHg propuesta por la Asociación Americana de Diabetes. El control glicémico fue significativamente peor en el grupo de adolescentes en comparación con el de adultos, sin embargo, esto no se tradujo en una mayor prevalencia de complicaciones crónicas en los primeros. Típicamente se ha descrito que las complicaciones crónicas de la diabetes mellitus se manifiestan durante el segundo decenio de hiperglicemia, lo cual resalta la importancia de la duración de la enfermedad en el desarrollo y progresión de las complicaciones asociadas. La mayor prevalencia de nefropatía y retinopatía diabéticas en la población de adultos podría ser atribuida, al menos en parte, a una mayor 
evolución de la enfermedad en relación con el grupo de adolescentes.

La mayor utilización de estatinas e inhibidores de la ECA por parte de los adultos diabéticos tipo 1 en relación con los adolescentes del estudio, es un reflejo de la mayor prevalencia de hipercolesterolemia y nefropatía diabética en los primeros. Un dato alarmante fue el hallazgo de que menos de la mitad de los sujetos del estudio alcanzaron el nivel meta de LDL colesterol propuesto por la Asociación Americana de Diabetes $(\mathrm{LDL}<100 \mathrm{mg} / \mathrm{dl}$ en $37.4 \%$ de pacientes). Incluso hubo una tendencia no significativa a un mayor porcentaje de adultos no alcanzando metas de LDL colesterol en comparación con los adolescentes $(\mathrm{P}=0.536)$.

La importancia de alcanzar la cifra meta de LDL colesterol, propuesto como objetivo primario del tratamiento de dislipidemia por el Tercer Reporte del Programa Nacional de Educación en Colesterol (NCEP) ATP III ${ }^{(11)}$ en pacientes con triglicéridos inferiores a $500 \mathrm{mg} / \mathrm{dl}$, radica en la asociación continua y logarítmica entre los niveles de LDL colesterol y el riesgo relativo de enfermedad coronaria ${ }^{(12)}$. A pesar de que la evidencia sobre terapia hipolipemiante en diabéticos tipo 1 es limitada, el Estudio de Protección del Corazón (Heart Protection Study) (en el subgrupo de 5963 diabéticos aleatorizados a simvastatina $v s$ placebo) demostró una reducción de eventos cardiovasculares en el subgrupo de 600 diabéticos tipo 1 similar a lo observado en los diabéticos tipo 2 (sin embargo, el beneficio no alcanzó significancia estadística $)^{(33)}$. Sin embargo, se debe recordar que en este estudio se incluyó diabéticos de más de 40 años de edad con un factor de riesgo cardiovascular adicional, por lo que no podríamos extrapolar los datos a la población evaluada en el presente estudio. En general, la Asociación Americana de Diabetes propone las mismas metas hipolipemiantes en diabéticos tipo $1 \mathrm{y}$ tipo $2^{(13)}$.

Uno de los hallazgos más relevantes del estudio fue la demostración de un peor control glicémico en la población de adolescentes en relación con la de adultos diabéticos tipo 1. Se ha demostrado que la hiperglicemia es un mediador clave del riesgo cardiovascular de diabéticos tipo 1. Ésta lleva a disfunción endotelial, rigidez de paredes vasculares arteriales, incremento en el grosor íntima media, neuropatía autonómica cardíaca y disfunción ventricular izquierda ${ }^{(21-23,27)}$. El estudio Oslo demostró que cada $1 \%$ de incremento en $\mathrm{HbAlc}$ se relacionó con un incremento de un $6.4 \%$ en el área de estenosis coronaria, determinado por ultrasonido intravascular (18 años de seguimiento $)^{(28)}$. El control glicémico estricto en comparación con el convencional en diabéticos tipo 1 , ha demostrado retrasar el desarrollo y progresión de la neuropatía cardíaca autonómica y mejorar la masa y función ventricular izquierdas ${ }^{(22,29-31)}$.

Actualmente existe evidencia a partir de estudios clínicos prospectivos aleatorizados utilizando puntos finales clínicos, de que el control glicémico estricto en la población de diabéticos tipo 1 retarda y reduce la progresión de complicaciones crónicas microvasculares (reducción de retinopatía en un 47\%, nefropatía clínica en un $54 \%$ y neuropatía en un $60 \%$; de acuerdo con el Estudio de Control y Complicaciones en Diabetes, $\left.\mathrm{DCCT}^{(19)}\right)$ y macrovasculares (reducción de cualquier evento cardiovascular en un $42 \%$ y reducción de infarto al miocardio no fatal, enfermedad cerebrovascular y muerte por enfermedad cardiovascular en un 57\%; de acuerdo con el estudio de Epidemiología de Intervenciones y Complicaciones en Diabetes, $\operatorname{EDIC}^{(20)}$ ), en comparación con el grupo recibiendo terapia convencional. Estos dos estudios resaltan la importancia de la optimización del control metabólico en el paciente diabético.

La exposición glicémica temprana en la evolución de la diabetes mellitus tipo 1 genera una impronta metabólica, conocida como memoria metabólica, que contribuye al riesgo cardiovascular. Un control glicémico estricto en las primeras etapas de la enfermedad reduce considerablemente el riesgo futuro de complicaciones macrovasculares. A parte del control glicémico temprano, la magnitud del descenso de la glicemia es otro factor que influye en la protección cardiovascular del diabético tipo 1. A diferencia de otros estudios en diabéticos tipo 1 en los que el control glicémico fue menos estricto, en el DCCT se alcanzó una HbAlc cercana al 7\% (grupo intensivo), lo cual, varios años después, redujo marcadamente varias complicaciones macrovasculares ${ }^{(32)}$.

A pesar de que las diferentes guías sobre metas de tratamiento para los pacientes diabéticos enfatizan la importancia de un control glicémico 
adecuado, con meta de HbA1c $<7 \%$ en ausencia de hipoglicemia significativa, de acuerdo con los Estándares de Cuidado Médico en Diabetes 2010 de la Asociación Americana de Diabetes ${ }^{(13)}$, se sabe que en la práctica clínica es difícil alcanzar muchas de las metas. El control óptimo de la enfermedad es difícil de alcanzar en la mayoría de los pacientes diabéticos. En el estudio DCCT, en el que participó una población altamente seleccionada y motivada, dotada de educación y seguimiento, sólo un $5 \%$ de los pacientes mantuvo una concentración promedio de hemoglobina glicosilada en el rango normal ( $\leq$ $6.5 \%)^{(5)}$.

El manejo y seguimiento adecuados de la diabetes tipo 1 requiere un abordaje multidisciplinario, en el que interactúan el paciente, su familia y el equipo y centro de atención en salud. Cada uno de los elementos es fundamental en la definición de metas de tratamiento y medidas orientadas al control óptimo de la enfermedad. Existen múltiples factores que influyen en el control glicémico y desarrollo y progresión de complicaciones crónicas en diabéticos tipo 1 .

En una revisión (6) sobre el efecto de las barreras externas sobre el control de la diabetes mellitus, las mismas se dividieron en 2 grandes grupos: barreras del paciente (influencia del nivel socioeconómico, educación del paciente y presencia o ausencia de un seguro en salud) y barreras del sistema de atención en salud (acceso a servicios de atención en diabetes mellitus de calidad y actitud del médico hacia la enfermedad). En relación con las barreras del paciente, el Estudio Epidemiológico de Complicaciones de la Diabetes de Pittsburgh (por sus siglas en inglés EDC; estudio prospectivo de seguimiento de 658 diabéticos tipo 1 de inicio antes de los 17 años, edad media de 28 años y duración media de la enfermedad de 20 años), reveló que tanto los pacientes con bajo nivel socioeconómico como aquellos con menor nivel educativo reportaron con menor frecuencia buen estado de salud y tuvieron peor control glicémico en comparación con los diabéticos tipo 1 de mayor nivel socioeconómico y educativo (diferencias fueron estadísticamente significativas).

El acceso a servicios de salud de calidad en diabetes mellitus es una de las barreras de los sistemas de atención en salud. Estudios han revelado que más del $90 \%$ de las visitas médicas del diabético son atendidas por médicos de atención primaria, quienes se caracterizan por un menor uso de medidas preventivas y peor control glicémico de los pacientes en comparación con la población diabética atendida por médicos especialistas. La actitud del médico hacia la diabetes es otra de las barreras que influyen en el control de la enfermedad. Algunos estudios han revelado que los médicos de atención primaria consideran más complejo el tratamiento de la diabetes mellitus en comparación con otras enfermedades crónicas.

Además de las barreras externas capaces de influir en el control de la enfermedad, existen otros factores que pueden modificar el control glicémico en la población de diabéticos tipo 1 . Diversos estudios previos han analizado el efecto de la edad del diabético tipo 1 sobre el control glicémico, y la relación con trastornos psicosociales y complicaciones crónicas de la enfermedad.

En un estudio observacional prospectivo realizado en el Hospital Universitario de Toronto $^{(4)}$, con 91 adolescentes femeninas diabéticas tipo 1 (edad promedio: $15 \pm 2$ años; duración promedio de enfermedad: $7 \pm 4$ años; seguimiento promedio: 4-5 años), se demostró una prevalencia basal de trastornos alimentarios del 29\%. De las pacientes sin trastornos alimentarios al inicio del estudio, el $15 \%$ desarrollaron alguno de estos trastornos durante el seguimiento. Hubo una correlación significativa directa entre la severidad del trastorno de la conducta alimentaria y el nivel de HbA1c, y entre la severidad del desorden alimentario al inicio del estudio y el desarrollo de retinopatía diabética 4 años después.

Dentro de los factores clínicos y de laboratorio presentes en la población de diabéticos tipo 1 al inicio del estudio de seguimiento DCCT/EDIC, asociados de forma significativa con el desarrollo de enfermedad cardiovascular, la edad fue uno de ellos. La edad mayor ( $31 \pm 6$ años) se asoció significativamente con el desarrollo de enfermedad cardiovascular en comparación con la edad menor ( $27 \pm 7$ años), independientemente del grupo de tratamiento al que fuera asignado el paciente $(\mathrm{P}<0.001)$. El estudio de seguimiento DCCT/EDIC ratifica el hecho de que el grupo etario de la población de diabéticos tipo 1 puede influir en el desarrollo de complicaciones 
crónicas de la enfermedad ${ }^{(7)}$. En el presente estudio no se documentó complicaciones crónicas macrovasculares, sin embargo sí hubo una relación entre el grupo etario y el desarrollo de complicaciones crónicas microvasculares (véanse párrafos anteriores).

En contraposición con los diabéticos tipo 1 con mejor control glicémico, aquellos con un peor control metabólico, presentan una mayor prevalencia de patologías psiquiátricas concomitantes, tienden a proceder de familias conflictivas y presentan una mayor predisposición a ausentarse de sus citas médicas de control ${ }^{(5)}$.

Desde que se establece el diagnóstico y durante el curso de la enfermedad, los niños y adolescentes diabéticos tipo 1 presentan una serie de crisis personales predecibles, manifestadas por sentimientos de tristeza intermitente, soledad, irritabilidad y aislamiento social. Los conflictos familiares al momento del debut se relacionan con problemas de adaptación temprana a la enfermedad, predisposición al desarrollo de trastornos de ansiedad y depresión, control glicémico subóptimo y asistencia irregular a las citas de seguimiento médico en niños y adolescentes diabéticos tipo $1^{(5)}$.

En un estudio que valoró el impacto de la inclusión de la psicología clínica en el manejo multidisciplinario de la diabetes, se demostró que ciertos factores psicosociales se relacionaron con pobre adherencia al tratamiento y peor control glicémico, algunos fueron: síndrome orgánico cerebral produciendo tendencia al olvido, reacciones de duelo por el fallecimiento de seres queridos, antecedente de abuso sexual o violación en la infancia, sentimientos encontrados por la separación marital o el divorcio de los padres y conflictos familiares por la diabetes ${ }^{(9)}$.

Uno de los factores que ha demostrado ser controversial en el control metabólico de los adolescentes diabéticos tipo 1 es la motivación intrínseca. Se cree que los adolescentes con mayor motivación intrínseca para el manejo de su enfermedad, podrían ser más persistentes en el cuidado de su diabetes y presentarían mejor control glicémico. En un estudio transversal con 43 adolescentes diabéticos tipo 1 (edad promedio $=14.14 \pm 1.73$ años; $65 \%$ mujeres; $74 \%$ caucásicos) se quiso poner a prueba la anterior hipótesis. El estudio reveló que los adolescentes con mayor motivación intrínseca reportaron una mayor adherencia al tratamiento $(\mathrm{r}=0.38, \mathrm{P}<$ 0.05 ), pero, sorprendentemente, tuvieron un peor control glicémico en comparación con el grupo con menor motivación intrínseca $(\mathrm{r}=0.48, \mathrm{P}<$ 0.05). Estos resultados han sido reportados previamente en la literatura, lo cual sugiere que los adolescentes encargados primariamente por el cuidado de su diabetes tienden a presentar pobre control glicémico. Los autores del estudio sugieren que los adolescentes diabéticos tipo 1 intrínsicamente motivados corren el riesgo de pobre control glicémico, probablemente por la toma de decisiones sobre el manejo de su enfermedad basados en su juicio propio, caracterizado por inexperiencia y desconocimiento sobre el manejo adecuado de la patología $^{(8)}$.

En el caso de la población de adolescentes es necesario considerar que es un grupo etario caracterizado por las aspiraciones al pensamiento y accionar independientes. Se recomienda la participación activa del adolescente en la toma de decisiones y definición de metas de tratamiento con el objetivo de incrementar la adherencia al tratamiento $^{(5)}$.

Los diabéticos tipo 1 presentan una mayor prevalencia de algunas patologías psiquiátricas en comparación con el resto de la población. Las 3 condiciones mayormente asociadas y que a su vez se relacionan con un peor control glicémico y mayor progresión de complicaciones crónicas, son la depresión, los trastornos de ansiedad y los trastornos alimentarios.

En el caso de la depresión, se ha demostrado que su prevalencia se encuentra aumentada en la población de adultos diabéticos tipo $1^{(5)}$. El efecto hiperglicemiante de la depresión parece depender de factores conductuales (conducta alimentaria, tabaquismo, actividad física, función cognitiva y adherencia al tratamiento) y factores fisiopatológicos (disregulación glucocorticoide, hiperactividad simpática y alteraciones en mediadores inflamatorios, que llevan a hiperglicemia y/o resistencia a la insulina). Se ha demostrado que la depresión favorece la progresión de complicaciones crónicas micro y macrovasculares en la población de diabéticos y aumenta el riesgo de muerte prematura ${ }^{(10)}$. Algunos estudios han demostrado una asociación entre retinopatía diabética más severa en 
diabéticos deprimidos en relación con controles no deprimidos ${ }^{(14,15)}$. Existe evidencia de que el alivio de la depresión se acompaña de mejoría en el control glicémico y en la sensibilidad a la insulina en sujetos diabéticos tipos 1 y $2_{10}$.

La población femenina de diabéticas tipo 1 presenta mayor riesgo de trastornos de la alimentación tipo bulimia y anorexia nerviosa. Estas pacientes con frecuencia omiten las aplicaciones de insulina con el objetivo de perder peso. Esta conducta se ha relacionado con deterioro en el control glicémico y desarrollo acelerado de retinopatía diabética ${ }^{(5)}$.

Los síndromes de ansiedad se presentan con mayor frecuencia en la población de diabéticos tipo 1 en comparación con la población general, se han relacionado con pobre control glicémico y se pueden confundir con cuadros de hipoglicemia $^{(5)}$.

\section{CONCLUSIONES Y RECOMENDACIONES}

La diabetes mellitus tipo 1 es una enfermedad crónica de base predominantemente autoinmunitaria cuya prevalencia ha aumentado en los últimos 20 años. Con frecuencia el debut y gran parte de la evolución de la patología se presentan durante la niñez y/o adolescencia, siendo ésta última una etapa de la vida caracterizada por las aspiraciones al pensamiento y accionar independientes. Uno de los principales hallazgos del presente estudio fue la demostración de un control glicémico significativamente peor en la población de adolescentes en comparación con la de adultos diabéticos tipo 1 de la Consulta Externa de Endocrinología del HSJD. A pesar de que no se analizó los factores específicos responsables de esta diferencia, gran parte de la misma podría ser atribuible a factores psicosociales.

Actualmente, varios proveedores de cuidados en diabetes reconocen la importancia de la inclusión de servicios de apoyo psicosocial capacitado en el manejo multidisciplinario de la diabetes. El Programa de Reconocimiento en Educación de la Asociación Americana de Diabetes apoya esta inclusión ${ }^{(9)}$.

\section{REFERENCIAS BIBLIOGRÁFICAS}

1. Diabetes Board-LA. Latino American Diabetes Education Board. 1997.

2. Gale E. Perspectives in Diabetes The Rise of Childhood Type 1-Diabetes in the 20th Century. Diabetes 2002;51:3363-3371.

3. Kasper D Braunwald E Fauci A Hauser S Longo D Jameson J. Harrison Principios de Medicina Interna. 15. edición, editorial McGraw-Hill Interamericana. México. 2001:37-43.

4. Rydall AC Rodin GM Olmsted MP Devenyi RG Daneman D. Disordered Eating Behavior And Microvascular Complications In Young Women With Insulin-Dependent Diabetes Mellitus. N Engl J Med 1997;336:1849-1854.

5. M. Jacobson. The Psychological Care Of Patients With Insulin-Dependent Diabetes Mellitus. N Engl J Med 1996;334:1249-1253.

6. Zgibor J Songer T. External Barriers to Diabetes Care: Addressing Personal and Health Systems Issues. Diabetes Spectrum 2001;14:23-28.

7. The Diabetes Control and Complications Trial/Epidemiology of Diabetes Interventions and Complications (DCCT/EDIC) Study Research Group. Intensive Diabetes Treatment and Cardiovascular Disease in Patients with Type 1 Diabetes. N Engl J Med 2005;353:2643-2653.

8. Greening L Stoppelbein L Moll G Palardy N Hocking M. Intrinsic Motivation and Glycemic Control in Adolescents with Type 1-Diabetes. Diabetes Care 2004;27(6):1517.

9. Leichter S Dreelin E Moore S. Integration of Clinical Psychology in the Comprehensive Diabetes Care Team. Clinical Diabetes 2004;22:129-131.

10. Leichter S See Y. Problems That Extend Visit Time and Cost in Diabetes Care: 1. How Depression May Affect the Efficacy and Cost of Care of Dia- 
betic Patients. Clinical Diabetes 2005;23:53-54.

11. Third Report of The National Cholesterol Education Program (NCEP) Expert Panel on Detection, Evaluation, and Treatment of High Blood Cholesterol in Adults (Adult Treatment Panel III).

12. Grundy SM Cleeman JL Merz CN et al. Implications of Recent Clinical Trials for the National Cholesterol Education Program Adult Treatment Panel III Guidelines. Circulation 2004;110(2):227-239.

13. American Diabetes Association. Standards of Medical Care in Diabetes-2010. Position Statement. Diabetes Care 2010;33:S11-S61.

14. Lustman PJ Griffith LS Clouse RE Cryer PE. Psychiatric illness in diabetes mellitus: relationship to symptoms and glucose control. J Nerv Ment Dis 1986;174:736-742.

15. Cohen $\mathrm{T}$ Welch $\mathrm{G}$ Jacobson AM de Groot M Samson J. The association of psychiatric illness and increased prevalence of retinopathy in patients with type 1 diabetes mellitus. Psychosomatics (in press).

16. Karvonen M Pitkaniemi J Tuomilehto J. The onset age of type 1-Diabetes in Finnish children has become younger. Diabetes Care 1999;22:1066-1070.

17. Gardner SG Bingley PJ Sawtell PA Weeks S Gale EA. Rising incidence of insulin dependent diabetes in children aged under-5 years in the Oxford region. BMJ 1997;315:713-717.

18. LaPorte RE Matsushima M Chang YF. Prevalence and incidence of insulindependent diabetes. In Diabetes in America $2^{\text {nd }}$ ed. National Institutes of Health, 1995;37-45 (NIH publ. no. 951468).

19. Diabetes Control and Complications Trial Research Group. The effect of intensive treatment of diabetes on the development and progression of long-term complications in insulin-dependent diabetes mellitus. N Engl J Med 1993;329:977-986.

20. Epidemiology of Diabetes Interventions and Complications Research Group. Protocol 1996. (Accessed November 23, 2005, at http://www.bsc.gwu.edu/bsc/studies/edi c.html.).

21. Libby $\mathrm{P}$ Nathan $\mathrm{DM}$ Abraham $\mathrm{K}$ et al. Report of the National Heart, Lung, and Blood Institute-National Institute of Diabetes and Digestive and Kidney Diseases Working Group on Cardiovascular Complications of Type 1 Diabetes Mellitus. Circulation 2005;111:34893493.

22. Boudina S Abel ED. Diabetic cardiomyopathy revisited. Circulation 2007;115:3213-3223.

23. Chan NN Vallance P Colhoun HM. Endothelium-dependent and-independent vascular dysfunction in type 1Diabetes: role of conventional risk factors, sex, and glycemic control. Arterioscler Thromb Vasc Biol 2003;23:10481054.

24. Larsen JR Brekke M Bergengen L et al. Mean HbAlc over 18 years predicts carotid intima media thickness in women with type 1-Diabetes. Diabetologia 2005;48:776-779.

25. Witte DR Tesfaye S Chaturvedi $\mathrm{N}$ et al. Risk factors for cardiac autonomic neuropathy in type 1 diabetes mellitus. Diabetologia 2005;48:164-171.

26. Shishehbor MH Hoogwerf BJ Schoenhagen $\mathrm{P}$ et al. Relation of hemoglobin Alc to left ventricular relaxation in patients with type 1 diabetes mellitus and without overt heart disease. Am J Cardiol 2003;91:1514-1517.

27. Larsen JR Sjoholm H Berg TJ et al. Eighteen years of fair glycemic control preserves cardiac autonomic function in type 1-Diabetes. Diabetes Care 2004;27:963-966. 
28. Larsen J Brekke M Sandvik L Arnesen H Hanssen KF Dahl-Jorgensen K. Silent coronary atheromatosis in type 1Diabetic patients and its relation to long-term glycemic control. Diabetes 2002;51:2637-2641.

29. The Diabetes Control and Complications Trial Research Group. The effect of intensive diabetes therapy on measures of autonomic nervous system function in the Diabetes Control and Complications Trial (DCCT). Diabetologia 1998;41:416-423.

30. Aepfelbacher FC Yeon SB Weinrauch LA D'Elia J Burger AJ. Improved glycemic control induces regression of left ventricular mass in patients with type 1 diabetes mellitus. Int $\mathrm{J}$ Cardiol 2004;94:47-51.

31. Weinrauch LA Burger A Gleason RE Lee AT D'Elia JA. Left ventricular mass reduction in type 1-Diabetic patients with nephropathy. J Clin Hypertens (Greenwich) 2005;7:159-164

32. Retnakaran R Zinman B. Type 1 diabetes, hyperglycaemia, and the heart. Lancet 2008;371:1790-1799.

33. Collins R Armitage J Parish S Sleigh P Peto R. Heart Protection Study Collaborative Group: MRC/BHF Heart Protection Study of cholesterol-lowering with simvastatin in 5963 people with diabetes: a randomized placebocontrolled trial. Lancet 2003;361:20052016.

\section{CONFLICTO DE INTERÉS}

En el presente trabajo no existió ningún conflicto de interés 